Rev. Mat. Iberoamericana 27 (2011), no. 3, 909-918

\title{
Steiner and Schwarz symmetrization in warped products and fiber bundles with density
}

\author{
Frank Morgan, Sean Howe and Nate Harman
}

\begin{abstract}
We provide very general symmetrization theorems in arbitrary dimension and codimension, in products, warped products, and certain fiber bundles such as lens spaces, including Steiner, Schwarz, and spherical symmetrization and admitting density.
\end{abstract}

\section{Introduction}

Symmetrization has always played a major role in geometry and analysis, especially for the isoperimetric problem, but it is hard to provide comprehensive statements and proofs. Steiner symmetrization in $\mathbb{R}^{N}$ replaces onedimensional slices with centered intervals. Schwarz symmetrization in $\mathbb{R}^{N}$ replaces $(N-1)$-dimensional slices with centered balls. Generalized Schwarz symmetrization in $\mathbb{R}^{N}$ replaces slices of some dimension $1 \leq n \leq N-1$ with centered balls. These results generalize readily to products $M \times \mathbb{R}^{n}$. Spherical symmetrization in $\mathbb{R}^{N}$ replaces slices by spheres about the origin with spherical caps.

Antonio Ros [15, Sect. 3.2] gave a beautiful proof of symmetrization in the context of manifolds with density. Our first Proposition 3 extends Ros to warped products as asserted by Morgan [11, Thm. 3.2] and is general enough to include spherical symmetrization (Rmk. 4) as well as Steiner and Schwarz symmetrization. Proposition 5 treats the smooth case with an analysis of when equality holds after Rosales et al. [17, Thm. 5.2]. Propositions 6 and 8 extend symmetrization to Riemannian fiber bundles with equidistant fibers in which horizontal movement from fiber to fiber preserves or scales volume.

2010 Mathematics Subject Classification: 49Q10, 53C12.

Keywords: Steiner symmetrization, Schwarz symmetrization, spherical symmetrization, warped products, fiber bundles, lens spaces, manifolds with density. 
Some simple examples are lens spaces (fibered by circles, Rmk. 7) as envisioned by Ros [16, 15, Thm. 2.11], similar Hopf circle fibrations of $\mathbb{S}^{2 n+1}$ over $\mathbb{C} \mathbb{P}^{n}$, the Hopf fibration of $\mathbb{S}^{7}$ by great $\mathbb{S}^{3} \mathrm{~S}$ and of $\mathbb{S}^{15}$ by great $\mathbb{S}^{7} \mathrm{~S}$. We were not, however, able to complete the proof by symmetrization envisioned by Vincent Bayle (private communication) to prove the still open conjecture that in $\mathbb{R}^{N}$ with a smooth, radial, log-convex density, balls about the origin are isoperimetric [17, Conj. 3.12], because horizontal movement from fiber to fiber does not preserve or scale volume.

Standard references on symmetrization are provided by Burago and Zalgaller [2, Sect.9.2] and Chavel [3, Sect.6]. Gromov [7, Sect.9.4] after [8, 5.A] provides some sweeping remarks and generalizations, including most of our results.

Acknowledgments. This work began with the Williams College, National Science Foundation SMALL undergraduate research Geometry Group at the University of Granada, Spain, summer 2009. We would like to thank Vincent Bayle, Antonio Cañete, Alexander Díaz, Rafael López, Manuel Ritoré, Antonio Ros, César Rosales, and David Thompson for help and inspiration.

\section{Symmetrization}

A convenient general definition of perimeter in a metric space with density is provided by Minkowski content or perimeter:

Definition 1. The Minkowski perimeter of a region $R$ is the lower right derivative

$$
\liminf _{\Delta r \rightarrow 0^{+}} \frac{\Delta V}{\Delta r}
$$

for $r$ enlargements. In a Riemannian manifold with continuous metric and density, the right limit $d V / d r$ exists and agrees with the usual definition of perimeter as long as the boundary of $R$ is rectifiable (see [6, Thm. 3.2.39]). We will use the following routine lemma ( $f$ in the source is the negative of our $f)$.

Lemma 2. (cf. [12, Lemma 2.4]) Let $f$, $h$ be real-valued continuous functions on $[a, b]$. Suppose that the upper right derivative of $f$ and the right derivative of $h$ satisfy

$$
\limsup _{\Delta x \rightarrow 0^{+}} \frac{f(x+\Delta x)-f(x)}{\Delta x} \leq \lim _{\Delta x \rightarrow 0^{+}} \frac{h(x+\Delta x)-h(x)}{\Delta x} .
$$

Then $f(b)-f(a) \leq h(b)-h(a)$. 
Proposition 3. (Symmetrization for warped products). Let $B, F$ be smooth, complete Riemannian manifolds. Consider a warped product $B \times{ }_{g} F$ with continuous metric $d s^{2}=d b^{2}+g(b)^{2} d t^{2}$ and continuous product density $\Phi(b) \cdot \Psi(t)$. Suppose that for some $p \in F$, geodesic balls about $p$ are isoperimetric. Let $R$ be a region of finite (weighted) perimeter. Then the Schwarz symmetrization $\operatorname{sym}(R)$, obtained by replacing the slice in each fiber by a ball about $p$ of the same (weighted) volume, has the same volume and no greater perimeter than $R$.

Remark. Although typically $F$ is $\mathbb{R}^{n}$ or $\mathbb{S}^{n}$, there are many other possibilities such as the paraboloid $\left\{z=x^{2}+y^{2}\right\}$ [1, Thms. 5, 8], [13, Thm. 3.1(A)]. Also balls about the origin may be replaced by half-planes $\left\{x_{n} \leq c\right\}$ (for $\mathbb{R}^{n-1} \times \mathbb{R}^{+}$as well as $\mathbb{R}^{n}$ ) when these have finite weighted volume. If $F$ is a space of revolution about $p$, for all balls about $p$ to be isoperimetric or even stationary (constant generalized mean curvature [10, §18.3]), the density $\Psi$ must be rotationally symmetric; for half-planes, $\Psi$ must be a function of $x_{1}, x_{2}, \ldots, x_{n-1}$ times a function of $x_{n}$.

Proof. The preservation of volume is just Fubini's theorem for warped products.

For small $r$, denote $r$-enlargements in $B \times{ }_{g} F$ by a superscript $r$ and $r$-enlargements in fibers by a subscript $r$. Consider a slice $\left\{b_{0}\right\} \times C=R\left(b_{0}\right)$ of $R$ and a ball about the origin $\left\{b_{0}\right\} \times D$ in the same fiber of the same weighted volume. For general $b$, consider slices $\left(\left\{b_{0}\right\} \times C\right)^{r}(b)$ of enlargements $\left(\left\{b_{0}\right\} \times C\right)^{r}$ of $\left\{b_{0}\right\} \times C$ and similarly slices $\left(\left\{b_{0}\right\} \times D\right)^{r}(b)$ of enlargements $\left(\left\{b_{0}\right\} \times D\right)^{r}$ of $\left\{b_{0}\right\} \times D$. If $C$ were a single point, then $\left(\left\{b_{0}\right\} \times C\right)^{r}(b)=$ $\{b\} \times C_{r^{\prime}}$ for some $r^{\prime}$ independent of $\mathrm{C}$, because the projection in $F$ of a shortest path $\gamma$ from $\left\{b_{0}\right\} \times C$ to a point in $\{b\} \times F$ is a shortest path $\gamma_{1}$ in $F$ and the length of $\gamma$ depends only on the length of $\gamma_{1}$. Hence for any $C$, $\left(\left\{b_{0}\right\} \times C\right)^{r}(b)=\{b\} \times C_{r^{\prime}}$ and $\left(\left\{b_{0}\right\} \times D\right)^{r}(b)=\{b\} \times D_{r^{\prime}}$ for the same $r^{\prime}$. Because the fiber density $\Psi(t)$ is independent of $b,\{b\} \times C$ and $\{b\} \times D$ have the same weighted volume. Since every $\{b\} \times D_{s}$ is isoperimetric for given volume, the lower right derivative $d V / d r$ for the family $C_{r}$ is at least as great as the right derivative $d V / d r$ for the family $D_{r}$, and hence the upper right derivative $d r / d V$ for the family $C_{r}$ is no greater than the right derivative $d r / d V$ for the family $D_{r}$. Consequently by Lemma 2 , if $C_{r_{1}}$ and $D_{r_{2}}$ have the same volume, $r_{1} \leq r_{2}$; conversely, when $r_{1}=r_{2}=r^{\prime}$, the volumes satisfy

$$
\left|\{b\} \times D_{r^{\prime}}\right| \leq\left|\{b\} \times C_{r^{\prime}}\right| \text {. }
$$

Therefore

$$
\left(\left\{b_{0}\right\} \times D\right)^{r}(b) \subseteq \operatorname{sym}\left(\left(\left\{b_{0}\right\} \times C\right)^{r}(b)\right) .
$$


Since this holds for all $b$,

$$
\left(\left\{b_{0}\right\} \times D\right)^{r} \subseteq \operatorname{sym}\left(\left(\left\{b_{0}\right\} \times C\right)^{r}\right) .
$$

Since this holds for all $b_{0}$,

$$
(\operatorname{sym}(R))^{r}=\bigcup_{b_{0}}\left(\left\{b_{0}\right\} \times D\right)^{r} \subseteq \bigcup_{\mathrm{b}_{0}} \operatorname{sym}\left(\left(\left\{b_{0}\right\} \times C\right)^{r}\right) \subseteq \operatorname{sym}\left(R^{r}\right) .
$$

Consequently,

$$
\left|(\operatorname{sym}(R))^{r}\right| \leq\left|R^{r}\right|
$$

and $\operatorname{sym}(R)$ has no more perimeter than $R$, as desired.

Remark 4. (Spherical symmetrization). In Proposition 3, at least for regions of finite volume, one may allow singular fibers as long as the union $S$ of such fibers has codimension 1 measure 0 . An important example is viewing $\mathbb{R}^{n+1}$ as the warped product $\{b \geq 0\} \times_{b^{n}} \mathbb{S}^{n}$ with singular fiber $\{b=0\}$, yielding so-called spherical symmetrization, using spherical caps to replace slices by spheres about the origin. To prove this generalization of Proposition 3, suppose that there were a counterexample. Then its restriction to the complement of an appropriate small $r$-neighborhood of the singular set $S$ would be a counterexample to the proof of Proposition 6. Note that the symmetrization of the restriction is just the restriction of the symmetrization. Additional perimeter introduced by truncation is negligible for most small $r$ by the finite volume hypothesis [10, §4.11].

Proposition 5 provides more general symmetrization with uniqueness for regions (typically isoperimetric regions) which satisfy certain smoothness hypotheses, as in the proof by Rosales et al. [17, Thm. 5.2] that in $\mathbb{R}^{n}$ with density $e^{r^{2}}$, balls about the origin uniquely minimize perimeter for given volume. For a special case, see Ritoré [14, § 1.3.2]; also see Monti [9]. Chlebík et al. [4] provide in Euclidean space an analysis of uniqueness without such smoothness hypotheses. Proposition 5 does not depend on Proposition 3.

Proposition 5. (Smooth case with uniqueness). Let $B, F$ be smooth Riemannian manifolds. Consider a smooth warped product $B \times{ }_{g} F$ with metric $d s^{2}=d b^{2}+g(b)^{2} d t^{2}$ and product density $\Phi(b) \cdot \Psi(t)$. Suppose that for some $p \in F$, geodesic balls about $p$ are isoperimetric. Let $R$ be a measurable set in $B \times{ }_{g} F$. Suppose that its topological boundary $\partial R$ meets almost every fiber smoothly (or not at all). Let $R^{\prime}$ denote its Schwarz symmetrization. Suppose that $\partial R^{\prime}$ also meets almost every fiber smoothly and that its intersection with other fibers contributes nothing to the area of $\partial R^{\prime}$. Then $R^{\prime}$ has the same volume and no greater perimeter than $R$. If they have the same perimeter and balls about the origin are uniquely isoperimetric in $F$ (up to measure 0), then $R=R^{\prime}$ up to a set of measure zero. 
Proof. The preservation of volume is just Fubini's theorem for warped products.

Let $B_{0}$ be the set of points $b$ in $B$ for which $\partial R$ and $\partial R^{\prime}$ are both smoothly transverse to the fiber over $b$. By hypothesis, almost every fiber meets $\partial R$ and $\partial R^{\prime}$ smoothly. By Sard's Theorem, almost every fiber meets $\partial R$ and $\partial R^{\prime}$ transversely. Hence almost all points of $B$ lie in $B_{0}$. For now we consider $b \in B_{0}$. Let $0 \leq \theta<\pi / 2$ denote the angle that $\partial R$ makes with the horizontal; at each point of $\partial R$ let $v$ be the horizontal vector in that direction with magnitude $\tan \theta$. (If $\partial R$ is locally the graph of a function $f: B \rightarrow F$, then $v=\nabla f$, and the component of $v$ in any direction gives the rate of change of $f$ in that direction.) The analogous $v^{\prime}$ for $\partial R^{\prime}$ has constant magnitude in each fiber. Because the metric $g$ depends only on $b$ and the density is a product density, horizontal movement just scales volume. If we vary b, any additional change in volume is due to $v$. Since corresponding slices have the same volumes, these changes must be the same in $R_{b}$ and $R_{b}^{\prime}$ :

$$
\int_{\partial R_{b}} \pm v=\int_{\partial R_{b}^{\prime}} v^{\prime}
$$

where the \pm sign depends on the local orientation of $\partial R_{b}$. The element of area $d A$ of $\partial R$ satisfies

$$
d A=\int_{\partial R_{b}} \sqrt{1+v^{2}} d b
$$

By Jensen's Theorem and the convexity of the function $h(x)=\sqrt{1+x^{2}}$,

$$
\int_{\partial R_{b}} \sqrt{1+v^{2}} \geq \sqrt{1+\left(\hat{\int}_{\partial R_{b}}|v|\right)^{2}}
$$

where the caret over the integral sign indicates normalization by the measure of the domain of integration. Let $\rho$ denote the ratio of the areas of $\partial R_{b}^{\prime}$ and $\partial R_{b}$. By hypothesis $\rho \leq 1$. By (2.1),

$$
\sqrt{1+\left(\hat{\int}_{\partial R_{b}}|v|\right)^{2}} \geq \sqrt{1+\left(\rho \hat{\int}_{\partial R_{b}^{\prime}}\left|v^{\prime}\right|\right)^{2}} \geq \rho \sqrt{1+\left(\hat{\int}_{\partial R_{b}^{\prime}}\left|v^{\prime}\right|\right)^{2}},
$$

because $h(x)=\sqrt{1+x^{2}}$ satisfies $h(\rho x) \geq \rho h(x)$ for any $0 \leq \rho \leq 1$, with equality only if $\rho=1$. Since $\left|v^{\prime}\right|$ is constant on $\partial R_{b}^{\prime}$,

$$
\sqrt{1+\left(\int_{\partial R_{b}^{\prime}}\left|v^{\prime}\right|\right)^{2}}=\hat{\int}_{\partial R_{b}^{\prime}} \sqrt{1+v^{\prime 2}}
$$


Finally,

$$
\int_{\partial R_{b}^{\prime}} \sqrt{1+v^{\prime 2}} d b=d A^{\prime} .
$$

Assembling inequalities (2.2)-(2.6) yields

$$
d A \geq d A^{\prime},
$$

with equality only if $\rho=1$. Integration yields

$$
|\partial R| \geq \int_{B_{0}} d A \geq \int_{B_{0}} d A^{\prime}=\left|\partial R^{\prime}\right|
$$

because by hypothesis the slices over $B_{0}$ include almost all of the area of $\partial R^{\prime}$. If equality holds, then for almost all slices, $d A=d A^{\prime}, \rho=1$, and by the uniqueness hypothesis, $R_{b}=R_{b}^{\prime}$ (up to measure 0). Almost all other slices are empty. Consequently $R=R^{\prime}$ up to a set of measure zero.

Remark. If we assume for example that $\partial R$ and $\partial R^{\prime}$ are smooth, then it follows that $R=R^{\prime}$.

The following proposition provides for certain fiber bundles associated to Riemannian submersions a similar symmetrization in a related warped product. A Riemannian submersion $\pi: M \rightarrow B$ has the property that $d \pi$, restricted to the orthogonal complement of its kernel, is an isometry. It follows that fibers are equidistant and that locally parallel transport normal to one fiber yields a diffeomorphism with any nearby fiber, which we assume preserves or scales volume.

Proposition 6. (Symmetrization for fiber bundles) Consider a smooth Riemannian submersion $M \rightarrow B$ with density $\Phi$ and a smooth warped product $B \times{ }_{g} F$ with product density $\Phi^{\prime}$. Assume that geodesic balls about a fixed point $p$ in $F$ are isoperimetric in every fiber of $B \times{ }_{g} F$, with no more perimeter than any competitor in the corresponding fiber of $M$. Further assume that parallel transport normal to the fibers from $M_{b_{1}}$ to $M_{b_{2}}$ scales volume on the fibers by $\left(g\left(b_{2}\right) / g\left(b_{1}\right)\right)^{n}$. Suppose that $M$ is compact or more generally that:

(1) $B$ is compact or more generally has positive injectivity radius and

(2) for some $r_{0}>0$, for $r<r_{0}$ the $r$-tube about a fiber $M_{b}$ under parallel translation from that fiber has metric

$$
d s^{2}=(1+o(1))\left(d b^{2}+d t^{2}\right)
$$

Here $d b$ is in the direction of parallel translation; $d t$, which depends on $b$, is the metric along fibers; and o(1) approaches 0 as $r$ approaches 0 , uniformly in $b$. 
Let $R$ be a region of finite perimeter. Consider the Schwarz symmetrization $\operatorname{sym}(R)$ in the warped product $B \times{ }_{g} F$, which replaces the slice of $R$ in each fiber with a ball about $p$ of the same volume in the corresponding fiber of $B \times{ }_{g} F$. Then $\operatorname{sym}(R)$ has the same volume and no greater perimeter than $R$.

Remark. For the simplest lens spaces (see Rmk. 7), $M=\mathbb{S}^{3}$ and $M_{b}=$ $F=\mathbb{S}^{1}$. In general, Gromov [7, Sect. 9.4$]$ suggests taking $F$ to be $\mathbb{R}$ or $\mathbb{R}^{+}$ with an appropriate density for which balls about $p=0$ are isoperimetric to reduce a product of well-understood factors to two dimensions.

Proof. The preservation of volume is just Fubini's theorem for Riemannian submersions.

As in the proof of Proposition 3 denote $r$-enlargements in $M$ by a superscript $r$ and $r$-enlargements in fibers by a subscript $r$. Let $r$ be a small positive number less than both $r_{0}$ and the injectivity radius of $B$. Consider a slice $C=R\left(b_{0}\right)$ of $R$ and a ball $D$ of the same volume about the origin in the corresponding fiber of $B \times{ }_{g} F$. For general $b$, let $C^{\prime}$ denote the image of $C$ in $M_{b}$ under normal parallel transport and let $D^{\prime}$ denote the copy of $D$ in $\{b\} \times{ }_{g} R^{n}$. Such horizontal movement just scales volume, in $M$ by hypothesis and in $B \times{ }_{g} F$ because the density is by hypothesis a product density. Therefore $\left|C^{\prime}\right|=\left|D^{\prime}\right|$. As in the proof of Proposition 3, $\left|D^{r}(b)\right|=\left|D_{r^{\prime}}^{\prime}\right|$, but due to the twisting in fiber bundles, it is not necessarily true that $\left|C^{r}(b)\right|=\left|C_{r^{\prime}}^{\prime}\right|$. By the uniformity hypothesis (2), the map by parallel transport based at $M_{b_{0}}$ from $B \times{ }_{g} F$ to $M$ distorts the metric by $1+o(1)$, uniform over $M$. Therefore

$$
C_{r^{\prime}}^{\prime} \subseteq C^{r+o(r)}(b)
$$

Since by hypothesis each $D_{r^{\prime}}^{\prime}$ has no more perimeter than any competitor in the corresponding fiber of $M$, the lower right derivative $d V / d r$ for the family $C_{r^{\prime}}^{\prime}$ is at least as great as the right derivative $d V / d r$ for the family $D_{r^{\prime}}^{\prime}$, and hence the upper right derivative $d r / d V$ for the family $C_{r^{\prime}}^{\prime}$ is no greater than the right derivative $d r / d V$ for the family $D_{r^{\prime}}^{\prime}$. Consequently by Lemma 2 , if $C_{r_{1}}$ and $D_{r_{2}}$ have the same volume, $r_{1} \leq r_{2}$; conversely, when $r_{1}=r_{2}=r^{\prime}$, the volumes satisfy

$$
\left|D_{r^{\prime}}^{\prime}\right| \leq\left|C_{r^{\prime}}^{\prime}\right| .
$$

Hence

$$
D^{r}(b) \subseteq \operatorname{sym}\left(C^{r+o(r)}(b)\right) .
$$

Since this holds for all $b$,

$$
D^{r} \subseteq \operatorname{sym}\left(C^{r+o(r)}\right) .
$$


Since this holds for all $b_{0}$,

$$
(\operatorname{sym}(R))^{r}=\bigcup_{b_{0} \in R} D^{r} \subseteq \bigcup_{b_{0} \in R} \operatorname{sym}\left(C^{r+o(r)}\right) \subseteq \operatorname{sym}\left(R^{r+o(r)}\right) .
$$

Consequently, $\left|(\operatorname{sym}(R))^{r}\right| \leq\left|R^{r+o(r)}\right|$, and $\operatorname{sym}(R)$ has no more perimeter than $R$, as desired.

Remark 7. As in Remark 4, at least for regions of finite volume, one may allow singular fibers where the projection fails to be a submersion, as long as the union $S$ of such fibers has codimension 1 measure 0 . An example is the fibration of $\mathbb{S}^{3}$ in $\mathbb{C}^{2}$ by orbits of the action mapping $\left(z_{1}, z_{2}\right)$ to $\left(e^{i k \theta} z_{1}, e^{i l \theta} z_{2}\right)$. If $k=l=1$, this is just the smooth Hopf fibration, but for general integers $k, l$ the orbits through $(1,0)$ or $(0,1)$ are singular. These circle fibrations lift to the lens spaces and generalize to all odd dimensions.

Similarly the uniformity hypothesis (1) on $B$ is not necessary. The second uniformity hypothesis (2) probably is not necessary (see Prop. 8), but our method of proof seems to need it. Of course if $R$ is compact then hypotheses (1) and (2) are not necessary.

In the unwarped case, including the lens spaces, the $1+o(1)$ factor in the proof is unnecessary, because distance from a point in a fixed fiber in the lens space is no greater than its value in the product (see [5, Prop. 8.6]).

Another interesting example is the cone over an $n$-dimensional Riemannian manifold $M_{0}$, which can be viewed as a warped product $\{b \geq 0\} \times_{b^{n}} M_{0}$. If regions in $M_{0}$ have no less perimeter than balls of the same volume in $S^{n}$, the cone can be compared to $\{b \geq 0\} \times{ }_{b^{n}} \mathbb{R}^{n}=\mathbb{R}^{n+1}[11, \S 3]$.

The following proposition relaxes the uniformity hypotheses of Proposition 6 for smooth regions and provides a framework for the analysis of when equality holds.

Proposition 8. (Smooth case for fiber bundles). Consider a smooth Riemannian submersion $M \rightarrow B$ with density and a smooth warped product $B \times{ }_{g} F$ with product density. Assume that geodesic balls about a fixed point $p$ in $F$ are isoperimetric in every fiber of $B \times{ }_{g} F$, with no more perimeter than any competitor in the corresponding fiber of $M$. Further assume that parallel transport normal to the fibers from $M_{b_{1}}$ to $M_{b_{2}}$ scales volume on the fibers by $\left(g\left(b_{2}\right) / g\left(b_{1}\right)\right)^{n}$. Let $R$ be a measurable set in $M$. Suppose that its topological boundary $\partial R$ meets almost every fiber smoothly (or not at all). Let $R^{\prime}$ denote its Schwarz symmetrization in the warped product $B \times{ }_{g} F$, which replaces the slice of $R$ in each fiber with a ball about $p$ of the same volume. Suppose that $\partial R^{\prime}$ also meets almost every fiber smoothly and that its intersection with other fibers contributes nothing to the area of $\partial R^{\prime}$. Then $R^{\prime}$ has the same volume and no greater perimeter than $R$. 
Remark. One may allow singular fibers, as long as the union of such fibers has codimension 1 measure 0 . In cases where there is always twisting, as in the lens space examples of Remark 7, if $R^{\prime}$ has the same perimeter as $R$, then $R$ must be a union of fibers.

Proof. The proof is almost identical to the proof of Proposition 5. We cannot hypothesize that $M$ has a product density, so we have added the hypothesis that parallel transport scales volume. Equation (2.2) becomes a (favorable) inequality due to the twisting in the fiber bundle. Everything else remains the same.

Note added in proof. More generally isoperimetric uniqueness follows from uniqueness in slices as shown by a Betta-Brock-Mercaldo-Posteraro preprint "On isoperimetric inequalities with respect to infinite measures" using piecewise smooth (polyhedral) approximations after De Giorgi and by standard geometric measure theory as in a Chlebik-Cianchi-Fusco paper [4] and with density in a Cianchi-Fusco-Maggi-Pratelli paper "On the isoperimetric deficit in Gauss space". This approach provides the easiest and most natural proof of uniqueness even in Gauss space, where an isoperimetric slice must be one of the two half-lines with the given volume.

\section{References}

[1] Benjamini, I. and CaO, J.: A new isoperimetric comparison theorem for surfaces of variable curvature. Duke Math. J. 85 (1996), 359-396.

[2] Burago, Yu. D. and Zalgaller, V. A.: Geometric inequalities. Die Grundlehren der mathematischen Wissenschaften 285. Springer-Verlag, 1988.

[3] Chavel, I.: Riemannian geometry. A modern introduction. Cambridge Studies in Advanced Mathematics 98. Cambridge University Press, 2006.

[4] Chlebímk, M., Cianchi, A. And Fusco, N.: The perimeter inequality under Steiner symmetrization: cases of equality. Ann. of Math.(2) 162 (2005), 525-555.

[5] Díaz, A., Harman, N., Howe, S. and Thompson, D.: Isoperimetric problems in sectors with density. To appear in Adv. Geom.

[6] Federer, H.: Geometric measure theory. Die Grundlehren der mathematischen Wissenschaften 153. Springer-Verlag, 1969.

[7] Gromov, M.: Isoperimetry of waists and concentration of maps. Geom. Funct. Anal. 13 (2003), 178-215.

[8] Gromov, M.: Spectral geometry of semi-algebraic sets. Ann. Inst. Fourier (Grenoble) 42 (1992), 249-274.

[9] Monti, R.: Heisenberg isoperimetric problem. The axial case. Adv. Calc. Var. 1 (2008), 93-121. 
[10] Morgan, F.: Geometric measure theory. A beginner's guide. Fourth edition. Elsevier/Academic Press, Amsterdam, 2009.

[11] Morgan, F.: In polytopes, small balls about some vertex minimize perimeter. J. Geom. Anal. 17 (2007), 97-106.

[12] Morgan, F.: Isoperimetric estimates in products. Ann. Global Anal. Geom. 30 (2006), 73-79.

[13] Morgan, F., Howards, H. and Hutchings, M.: The isoperimetric problem on surfaces of revolution of decreasing Gauss curvature. Trans. Amer. Math. Soc. 352 (2000), 4889-4909.

[14] Ritoré, M.: Geometric flows, isoperimetric inequalities and hyperbolic geometry. In Mean curvature flow and isoperimetric inequalities, 45-113. Adv. Courses Math. CRM Barcelona. Birkhäuser Verlag, Basel, 2010.

[15] Ros, A.: The isoperimetric problem. In Global theory of minimal surfaces (Proc. Clay Math. Inst. Summer School, 2001), 175-209. Clay Math. Proc. 2. Amer. Math. Soc., Providence, RI, 2005.

[16] Ros, A.: The isoperimetric problem for lens spaces, in preparation.

[17] Rosales, C., Cañete, A., Bayle, V. and Morgan, F.: On the isoperimetric problem in Euclidean space with density. Calc. Var. Partial Differential Equations 31 (2008), 27-46.

Recibido: 25 de noviembre de 2009

Frank Morgan

Department of Mathematics and Statistics

Williams College

Williamstown, MA 01267, USA

Frank.Morgan@williams.edu

Sean Howe

Institute of Mathematics

Leiden University

Leiden, The Netherlands

seanpkh@gmail.com

Nate Harman

Department of Mathematics and Statistics

University of Massachusetts

Amherst, MA 01003, USA

nateharman12340yahoo.com

We acknowledge partial support by the National Science Foundation (grants to Morgan and to the SMALL REU), the Spanish Ministerio de Educación y Ciencia (grant to Ritoré, Rosales, et al.), the University of Granada, and Williams College. 